\title{
EFFECTS OF REHABILITATION SERVICES ON ANXIETY, DEPRESSION, CARE-GIVING BURDEN AND PERCEIVED SOCIAL SUPPORT OF STROKE CAREGIVERS
}

\author{
Ali Yavuz Karahan', Sami Kucuksen², Halim Yilmaz', Ali Salli², Tayfun Gungor², Muhammed Sahin ${ }^{2}$
}

The Department of Physical Medicine and Rehabilitation of State Hospital of Konya/Turkey ${ }^{1}$, The Department of Physical Medicine and Rehabilitation of Necmettin Erbakan University Faculty of Medicine Konya/Turkey ${ }^{2}$, The Department of Physical Medicine and Rehabilitation, Konya Education and Research Hospital, Konya, Turkey ${ }^{3}$

Summary: Background: Few data are available on the specific care giving-related problems of stroke patient's caregivers and factors that influence the burden of these caregivers. Aim: To study the influences of the active rehabilitation process on anxiety, depression, care burden and perceived social support level of stroke patients caregivers. Design: A prospective clinical trial. Setting: Patients and caregivers entering a rehabilitation program at a university hospital in Turkey. Populations: Ninety patients with a first episode of stroke and 90 caregivers responsible for their care were recruited for our study. Methods: Patients and caregivers were assessed before and after the active rehabilitation process. The functional disability level of the patients was assessed by Functional Independence Measure (FIM). The Beck Anxiety Scale (BAS) and the Beck Depression Scale (BDS) were used for anxiety and depression assessment, the Zarit Care Burden Scale (ZCBS) for care burden assessment and the Multi-Dimensional Scale of Perceived Social Support (MDSPSS) for perceived social support assessment. Results: A statistically significant rise is observed in the special person sub-assessment of MDSPSS in both female and male caregivers. Also, a significant decrease in care burden, anxiety and depression levels of caregivers was noted after the rehabilitation program $(\mathrm{p}<0.05)$. Conclusion: Caregivers accept the rehabilitation period as important social support in addition to the support provided by family and friends. Also, our positive results were associated with an improvement in the patients' functional level and an increase in the acquisition of knowledge and skill required of caregivers in order to provide care during rehabilitation. Clinical Rehabilitation Impact: The rehabilitation team should be aware of the fact that the perceived care burden may be greater due to the lack of knowledge concerning available resources and due to the inability to cope with stress effectively.

Key words: Rehabilitation; Stroke; Caregiver; Depression; Social support

\section{Introduction}

Stroke rehabilitation is becoming increasingly important and the lives of patients are being facilitated with functional outcomes as life expectancy has increased. In recent years, successful results have been achieved in stroke treatment (1, 2). However, the majority of patients who survive a stroke are only able to continue their daily activities by being dependent on others. Patients and their families may experience a variety of physical, psychological, social, economic, and spiritual problems $(2,3)$. Care service that is provided by caregivers (CG) to stroke patients (SP) involves health care (buying medication, treatment, monitoring etc.), personal care (bathing, feeding, toilet, dressing etc.), coordination of social services received by the patient, shopping, housework, money management, physical aid, and sometimes sharing the same house (4). Negative objective and subjective outcomes such as psychological problems, physical health problems, economic and social problems, disturbed family affairs, feelings of not having control of issues arising from all these care services are defined as "care burden" $(4,5)$. Care services, which are often provided by one of the family members, can lead to negative results and hardships in addition to the positive outcomes of increased love and sincerity with the patient, personal development, development of close affairs, social support, self-respect and psycho-social satisfaction (4-6).

Approximately $80 \%$ of SP are able to return to the community with dependence on the emotional and social support of family members after the initial hospitalization and stroke rehabilitation $(1,2)$. The purpose of stroke rehabilitation is to equip the SP with the physical, psychological, social, vocational and educational potential to the extent permitted by physiological or anatomical disorders and environmental restrictions (1-3). In the rehabilitation period, a training and problem solving process aiming at decreasing the rate of disability is experienced by focusing on the condition. While the disabled patient was medically assisted in the focus of 
the process, the caregivers looking after the patient play a role within the process and are seriously affected by such a process $(5,7)$.

The purpose of our study was to study the influences of the active rehabilitation process on anxiety, depression, care burden and the perceived social support level of the CG.

\section{Patients and Method}

After approval by the Ethics Committee, 90 SPs and their individual CGs were recruited for our study while undergoing in-patient rehabilitation at Necmettin Erbakan University Medical Faculty Physical Medicine and Rehabilitation Department. Written consent of SPs and CGs was obtained.

The study inclusion criteria were:

- hemiplegia after stroke must be unilateral,

- minimum 1 month, maximum 6 months must have elapsed after stroke,

- care of the SP must be assumed by one single CG,

- CG must be a family member, not a professional caregiver,

- CG must not have a previously diagnosed psychological disorder.

Three SPs were withdrawn from the study because of recurrent stroke during rehabilitation, while 5 additional SPs were dropped due to discharge for various reasons prior to completion of the rehabilitation period. Thus, the study was completed with 83 SPs and CGs.

The age and gender of SPs were recorded. The functional disability level of the patients was assessed before and after rehabilitation using a Turkish version of Functional Independence Measure (FIM) (8).

CGs were assessed before and after the rehabilitation process. The Beck Anxiety Scale (BAS) and the Beck Depression Scale (BDS) were used for anxiety and depression assessment, the Zarit Care Burden Scale (ZCBS) for care burden assessment and the Multi-Dimensional Scale of Perceived Social Support (MDSPSS) for perceived social support assessment.

FIM is a scale that assesses 18 activities involving selfcare, sphincter control, motion, communication and social perception (8). It is an ordinal scale composed of 18 items with 7 levels ranging from 1 (total dependence) to 7 (total independence). The FIM can be subdivided into a 13-item motor subscale and a 5-item cognitive subscale. The ranges of scoring for the motor and cognitive subscales are 13 to 91 and 5 to 35 , respectively. The total score is 126 . The patients were tested by a qualified physiatrist upon admission and discharge (8).

The BAS measures the frequency of the patients' anxiety symptoms. It is a Likert-type self-assessment scale consisting of twenty one items scored between 0-3. A high score indicates a high level of anxiety. It was developed by Beck et al. and the validity and reliability study in our country was performed by Ulusoy et al $(9,10)$. 0-7 points from the scale may be interpreted as minimal anxiety symptoms, 8-15 points as moderate, 16-25 points as medium, and 26-63 points as severe anxiety symptoms $(9,10)$.
The BDS measures physical, emotional and cognitive symptoms observed during depression. It is a self-assessment scale involving twenty-one symptom categories. The highest score is 63 . The higher the score, the more severe the depression is (11). It was developed by Beck et al. and the validity and reliability study in our country was performed by Hisli et al (12). 0-9 points from the scale may be interpreted as minimal depression symptoms, $10-16$ points as moderate, $17-29$ points as medium, and 30-63 points as severe depression $(12,13)$.

The ZCBS assesses the stress suffered by those who give care to the persons in need. The scale consists of 22 questions and has a Likert-type assessment ranging from 1 to 5 as "never", "rarely", "sometimes", "often" or "almost every time". The highest score is 110 while the lowest is 22 $(14,15)$. There is no data about the cutoff of the scale but a score between 22 and 46 may be interpreted as moderate burden, medium burden between 47-55, severe burden if 56 and over $(14,15)$. Questions in the scale are generally about social and emotional aspects, and high scores from the scale indicate that the care giver's burden is high $(4,5)$. A validity study for ZCBS was proved by Ozlu et al (15).

The adequacy of social support received from three different sources (family, friends and special persons) is assessed with the MDSPSS. This scale was developed by Zimet et al. and a Turkish validity and reliability study has been performed $(16,17)$. The scale involves twelve statements and includes three different supports: family, friends and special persons (doctor, relative, neighbor, etc.). Sub-dimension scores may be summed in order to calculate the total scale score. The score of sub-dimensions ranges between 4 and 28, while the total scale score ranges from 12 and 84 . A high score from the scale indicates that the perceived social support is high $(16,17)$.

An IBM Statistics 16.0 (SPSS) pack program was used for the statistical assessment of data. The suitability of recurrent variables to normal distribution was tested. The Mann-Whitney $U$ test was used to assess non-parametric independent variables. The Wilcoxon test was used to calculate the difference between medians of non-parametric recurrent variables. The T-test method in dependent groups was used to find the difference between averages of recurrent variables that provide parametric conditions. A statistically significant value was specified as $\mathrm{p}<0.05$.

\section{Results}

Of the 83 SPs who completed the study, $41 \%$ (34) were women and 59\% (49) were men. The average age was 62.2 \pm 10.4 years in female SPs and $64.3 \pm 9.1$ years in male SPs. The time that elapsed after stroke was $72.6 \pm 27.3$ days on average.

Asshown in table 1 there was significant improvement in post-rehabilitation cognitive, motor and total FIM values compared to pre-rehabilitation values $(\mathrm{p}<0.001)$ (Table 1). 
$77.1 \%$ (64) of CGs were women and $22.9 \%$ (19) were men. The whole demographic characteristics of the participants could be seen in Table 2 (Table 2).

There was no statistically significant difference between baseline ZCBS, BDS and BAS values of CGs in terms of gender (p: 0.311). Also, as shown in the Table 3, the post-rehabilitation reduction in all these three parameter values were statistically significant in females, males and total $(p<0.001)$ (Table 3).

In terms of gender, there was no statistically significant difference between baseline family, friend, special person support and total MDSPSS value of CGs (respectively p: $0.326,0.115,0.214,0.098)$. Also, as shown in the Table 3 , an increase in special person and total MDSPSS values was statistically significant in men, women and total (Special person support MDSPSS $\mathrm{p}<0.001$ ) (Total MDSPSS $\mathrm{p}<0.001)$ (Table 3).

Tab. 1: Functional independence levels of stroke patients before and after rehabilitation.

\begin{tabular}{|l|c|c|c|c|}
\hline $\begin{array}{l}\text { FIM } \\
\text { (n: 83) }\end{array}$ & $\begin{array}{c}\text { Before } \\
\text { Rehabilitation } \\
\bar{\chi} \pm \mathrm{Sd}\end{array}$ & $\begin{array}{c}\text { After } \\
\text { Rehabilitation } \\
\bar{\chi} \pm \mathrm{Sd}\end{array}$ & $\mathbf{t}$ & $\mathbf{P}$ \\
\hline Motor & $49.0 \pm 17.1$ & $56.5 \pm 17.4$ & 8.215 & $<0.001$ \\
\hline Cognitive & $22.4 \pm 7.1$ & $24.2 \pm 7.0$ & 8.290 & $<0.001$ \\
\hline Total & $71.4 \pm 23.0$ & $80.7 \pm 23.3$ & 8.126 & $<0.001$ \\
\hline
\end{tabular}

FIM:Functional Independence Measuremenrt, t: Value of 2 related samples test

Tab. 2: Demographic data of caregivers.

\begin{tabular}{|c|c|c|c|}
\hline Caregiver & $\begin{array}{c}\text { Male (n: 19) } \\
\quad \text { (\% 22.9) }\end{array}$ & $\begin{array}{c}\text { Female } \\
(\mathrm{n}: 64) \\
(\% \text { 77.1) }\end{array}$ & $\begin{array}{c}\text { General } \\
(\mathrm{n}: \mathrm{83}) \\
(\% \mathbf{1 0 0})\end{array}$ \\
\hline Age (year) & $51.6 \pm 12.8$ & $46.4 \pm 9.8$ & $47.6 \pm 10.7$ \\
\hline \multicolumn{4}{|l|}{ Relation } \\
\hline Spouse & $12(\% 63.2)$ & $17(\% 26.6)$ & $29(\% 34.9)$ \\
\hline $\begin{array}{l}\text { Daughter or } \\
\text { son }\end{array}$ & $7(\% 36.8)$ & $44(\% 68.8)$ & $51(\% 61.4)$ \\
\hline $\begin{array}{l}\text { Mother or } \\
\text { father }\end{array}$ & - & $2(\% 3.1)$ & $2(\% 2.4)$ \\
\hline Other & - & $1(\% 1.6)$ & $1(\% 1.2)$ \\
\hline \multicolumn{4}{|l|}{$\begin{array}{l}\text { Education } \\
\text { level }\end{array}$} \\
\hline İlliterate & - & $7(\%$ 10.9) & $7(\% 8.4)$ \\
\hline $\begin{array}{l}\text { Primary or } \\
\text { secondary } \\
\text { school }\end{array}$ & $3(\% 15.8)$ & $46(\% 71.9)$ & $49(\% 59.0)$ \\
\hline High school & $14(\% 73.7)$ & $10(\% 15.6)$ & $24(\% 28.9)$ \\
\hline University & $2(\% 10.5)$ & $1(\% 1.6)$ & $3(\% 3.6)$ \\
\hline $\begin{array}{l}\text { Care duration } \\
\text { (days) }\end{array}$ & $74.3 \pm 329.2$ & $70.9 \pm 26.5$ & $71.7 \pm 27.0$ \\
\hline
\end{tabular}

\section{Discussion}

The rehabilitation of SPs involves bringing the SP to a maximum level of physical, psychological, social and vocational independence, thus leading to an improvement in their quality of life $(3,7)$. As an important disease that restricts the physical capabilities of individuals, a stroke brings different responsibilities and burdens to CGs in addition to causing certain lifestyle changes in patients (4). CGs often do not have the knowledge and skill required for providing long-term care (4). They may feel uncertain as to how to access and use available resources in a situation never before encountered. This may lead to feelings of insecurity and unpreparedness due to the care burden that they have undertaken $(4,5)$.

It has been previously noted that the care burden of CGs is particularly associated with the dependence level and cognitive functions of SP, and the care burden is negatively influenced with an increase in care duration and time spent in patient-care. The CG's age and health condition is one of the factors affecting care burden $(7,18)$. Defining the care burden is the first thing to assess in order to decrease $\mathrm{CG}$ burden during the active rehabilitation process. Knowing and revealing this burden will contribute to increasing the quality of life of both the CGs and receivers $(18,19)$. Morimoto et al. studied CGs of hemiplegic patients after stroke, reporting that $71 \%$ of CGs were women. Depression symptoms had been detected and quality of life had decreased in $52 \%$ of CGs, and the care burden found with ZCBS increased in relation to the independence level of the patient (20). McCullagh et al. assessed 232 SPs and their CGs and found that $48 \pm 13.2$ care burden by using ZCBS on the $3 \mathrm{rd}$ month (5). The average ZCBS was found to be 33.02 \pm 15.92 by Mollaoglu et al. (20). In our study; the average ZCBS values were $49.1 \pm 6.2$ before rehabilitation and 41.0 \pm 2.9 after rehabilitation. The influence of the rehabilitation process on care burden of CGs was statistically significant $(\mathrm{p}<0.001)$.

CGs with a severe, chronic illness may experience emotional restlessness, insomnia, reduction in self-respect, social isolation, depression, increased alcohol and drug intake and difficulty in problem-solving (22). Anger, disappointment, self-condemnation, worry, and desperateness are among other emotional problems faced by CGs $(22,23)$. The most common symptoms are increased stress and anxiety. The CG may generalize the feeling of anxiety derived from care to his/her whole life. It has been previously shown that depression and anxiety levels in stroke CGs increased and depression was observed in 23-29\% of CGs $(22,25)$. Though studies report that the CG's level of depression is inversely proportional to the level of independence of the patient, there are studies reporting that depression is independent of the stroke severity $(22,25)$. It has also been reported that the increased level of depression in CGs may be related to female gender, older CGs, poor financial capability, existence of chronic diseases in the CG himself/herself and in cases 
Tab. 3: Care burden, depression, anxiety and perceived social support levels of caregivers before and after rehabilitation.

\begin{tabular}{|c|c|c|c|c|}
\hline & $\begin{array}{c}\text { Beginning of } \\
\text { rehabilitation } \\
\bar{\chi} \pm \mathrm{Sd}\end{array}$ & After rehabilitation & Value & $\mathbf{p}$ \\
\hline \multicolumn{5}{|c|}{ Zarit Caregiver Burden } \\
\hline Women (n: 64) & $49.0 \pm 6.1$ & $41.2 \pm 3.0$ & t: 9.81 & $<0.001$ \\
\hline Men (n: 19) & $49.3 \pm 6.5$ & $40.1 \pm 2.6$ & z: 3.59 & $<0.001$ \\
\hline General (n: 83) & $49.1 \pm 6.2$ & $41.0 \pm 2.9$ & t: 11.12 & $<0.001$ \\
\hline \multicolumn{5}{|c|}{ Beck Depression Scale } \\
\hline Women (n: 64) & $17.9 \pm 4.2$ & $13.1 \pm 3.0$ & t: 21.21 & $<0.001$ \\
\hline Men (n: 19) & $16.5 \pm 2.7$ & $12.0 \pm 2.5$ & z: 3.89 & $<0.001$ \\
\hline General (n: 83) & $17.6 \pm 3.9$ & $12.9 \pm 2.9$ & t: 21.75 & $<0.001$ \\
\hline \multicolumn{5}{|c|}{ Beck Anxiety Scale } \\
\hline Women (n: 64) & $11.5 \pm 3.1$ & $8.0 \pm 3.0$ & t: 17.53 & $<0.001$ \\
\hline Men (n: 19) & $10.8 \pm 2.4$ & $7.0 \pm 2.5$ & z: 3.75 & $<0.001$ \\
\hline General (n: 83) & $11.4 \pm 3.0$ & $7.7 \pm 2.9$ & t: 24.57 & $<0.001$ \\
\hline \multicolumn{5}{|c|}{ MDSPSS Family Support } \\
\hline Women (n: 64) & $19.0 \pm 6.0$ & $20.9 \pm 4.2$ & t: 2.18 & 0.331 \\
\hline Men (n: 19) & $19.3 \pm 6.5$ & $19.5 \pm 2.7$ & z: 0.19 & 0.190 \\
\hline General (n: 83) & $19.0 \pm 6.1$ & $20.6 \pm 3.9$ & t: 1.93 & 0.287 \\
\hline \multicolumn{5}{|c|}{ MDSPSS Friend Support } \\
\hline Women (n: 64) & $15.9 \pm 4.2$ & $16.5 \pm 3.2$ & $\mathrm{t}: 3.03$ & 0.112 \\
\hline Men (n: 19) & $14.5 \pm 2.7$ & $15.8 \pm 2.4$ & z: 1.72 & 0.097 \\
\hline General (n: 83) & $15.6 \pm 3.9$ & $16.4 \pm 3.0$ & t: 2.08 & 0.191 \\
\hline \multicolumn{5}{|c|}{ MDSPSS Special Person } \\
\hline Women (n: 64) & $14.5 \pm 3.2$ & $20.2 \pm 8.1$ & t: 7.58 & $<0.001$ \\
\hline Men (n: 19) & $13.8 \pm 2.4$ & $18.6 \pm 6.0$ & z: 3.40 & $<0.001$ \\
\hline General (n: 83) & $14.4 \pm 3.0$ & $19.8 \pm 7.6$ & t: 8.58 & $<0.001$ \\
\hline \multicolumn{5}{|l|}{ MDSPSS Total } \\
\hline Women (n: 64) & $49.5 \pm 10.1$ & $57,7 \pm 14,5$ & t: 10.89 & $<0.001$ \\
\hline Men (n: 19) & $47.8 \pm 7.3$ & $54.1 \pm 9.6$ & z: 3.48 & $<0.001$ \\
\hline General (n: 83) & $49.1 \pm 9.5$ & $56.9 \pm 13.6$ & t: 12.02 & $<0.001$ \\
\hline
\end{tabular}

MDSPSS: Multi Dimension Scale of Perceived Social Support

requiring care-giving to more than one person $(22,26)$. No difference was observed in our study between the levels of anxiety and depression of female and male CGs (BAS p: 0.121 BDS p: 0.112). A statistically significant improvement was recorded in the BAS and BDS values of CGs with an active rehabilitation process $(\mathrm{p}:<0.001)$.

Social support is widely posited as a valuable resource comprising tangible forms of assistance that individuals receive from family, friends and others. Clinicians have begun to recognize the importance of social support in the form of family and friends. Cultural networks have also identified the benefits of social support for individuals' health and wellbeing, including a lower incidence of mental and physical disorders, a decrease in the level of stress, a more positive adjustment to chronic disease, as well as other positive physical and psychological health benefits (27-29).

Rehabilitation is the process by which a stroke survivor and their caregiver works with a team of health care providers with the aim of regaining the function lost after a stroke to the highest possible extent $(5,29,30)$. By joining a comprehensive rehabilitation program, stroke survivors can maximize their chances of recovery and, in most cases, can regain a substantial portion of function lost due to the stroke $(4,26)$. Rehabilitation provides a social support for CGs as a way of coping with caregiver burden. One of the theoretical explanations claiming to explain the ef- 
fect of social support on coping with stress is the buffering model. The buffering model proposes that social support provides a buffer against stressful life events and is related to well-being primarily for people under stress $(4,26)$. According to this model, social support may intervene between a stressful event and the stress reaction by influencing the stress appraisal response. Social support may also alleviate the impact of stress appraisal by reappraisal, providing a solution, or by reducing the importance of the problem. The alternative main effects model proposes that social support has a beneficial effect irrespective of the individual's situation $(26,27)$. By meeting basic human needs for intimacy, a sense of belonging and reassurance of one's worth, social support enhances health and well-being regardless of stress levels $(27,28)$. According to the results of our study, a statistically significant rise is observed in special person sub-assessment of both female and male CGs MDSPSS, even though non-significance differences were found only in friend and family support sub-assessments of MDSPSS. This shows that CGs accept the rehabilitation period as an important social support in addition to the the support provided by family and friends.

\section{Conclusion}

Significant, functional acquisitions were achieved at the end of the active rehabilitation process according to our study results. A significant decrease in care burden, anxiety and depression levels in CGs of SPs was noted. These positive results in CGs were associated with an improvement in the SP's functional level, and in the CG's acquisition of knowledge and skill required to provide care during rehabilitation.

The rehabilitation team should be aware of the fact that the perceived care burden may be greater due to the lack of knowledge about available resources and due to the inability to cope with stress effectively. We believe that the recognition of efforts to reduce the negative effects of care burden on CGs as a part of the rehabilitation process will strengthen the social support aspect of rehabilitation.

\section{References}

1. Yildiz N, Sanal E, Sarsan A, Topuz O, Ardic F. Characteristics of stroke patient and the factors affecting their functional outcomes. J PMR Sci 2009; 12: 59-66.

2. Karatepe AG, Kaya T, Sen N, Günaydin R, Gedizlioglu M. The risk factors in patients with stroke and relations with functional independence. Turk J Phys Med Rehab 2007; 53: 89-93.

3. Bardak AN, Ersoy S, Akcan Z, et al. Functional outcome of inpatient stroke rehabilitation Turk J Phys Med Rehab 2008; 54: 17-21.
4. Atagun Mİ, Balaban ÖD, Atagün Z, Elagöz M, Özpolat A. Caregiver Burden in Chronic Diseases. Current Approaches in Psychiatry 2011; 3(3): 513-52.

5. McCullagh E, Brigstocke G, Donaldson N, Kalra L. Determinants of care giving burden and quality of life in caregivers of stroke patients. Stroke 2005; 36: 2181-86.

6. Dökmen ZY, The relations between mental health and perceived social support of family caregivers. Ankara University Journal of Institute of Social Sciences 2012; 3(1): 3-38.

7. Bergström AL, Eriksson G, von Koch L, Tham K. Combined life satisfaction of persons with stroke and their caregivers: associations with caregiver burden and the impact of stroke. Health Qual Life Outcomes 2011; 11(9): 1.

8. Kucukdeveci AA, Yavuzer G, Elhan AH, Sonel B, Tennant A. Adaptation of the Functional Independence Measure for use in Turkey. Clin Rehabil 2001; 15(3): 311-19.

9. Beck AT, Epstein N, Brown G, Steer RA. An inventory for measuring clinical anxiety: psychometric properties. J Consult Clin Psychol, 1988; 56: 893-97.

10. Ulusoy M, Sahin N, Erkman H. Turkish Version of The Beck Anxiety Inventory: psychometric Properties. J Cognitive Psychotherapy: Int Quaterly, 1998; 12: $28-35$

11. Beck AT, Ward CH, Mendelson M, Mock J, Erbaugh J. An inventory for measuring depression. Archives of General Psychiatry 1961; 4: 53-63.

12. Hisli, N. A study on the validity of the Beck depression inventory. Turkish Journal of Psychology 1987; 6: 118-22.

13. Karahan AY, Islam S. A comparative study on caregiver burden of caregivers to physically disabled, pediatric and geriatric patients. Journal of Marmara University Institute of Health Sciences 2013; 3(1): 1-7.

14. Zarit SH, Reever KE, Bach-Peterson J. Relatives of the impaired elderly: correlates of feelings of burden. Gerontologist 1980; 20: 649-55.

15. Ozlu A, Y1ldiz M, Aker T. A reliability and validity study on the Zarit caregiver burden scale. Archives of Neuropsychiatry 2009; 46: 38-42.

16. Zimet GD, Dahlen NW, Zimet SG, Farley GK. The Multidimensional Scale of Perceived Social Support. J Pers Assess 1988; 52: 30-41.

17. Eker D, Arkar H, Yaldız H. The factor structure, reliability and validity of the Multidimensional Scale of Perceived Social Support. Türk Psikiyatri Dergisi 2001; 12(1): 17-25.

18. Oliveira AR, Costa AG, Sousa VE, Araujo TL, Silva VM, Lopes MV, et al. Scales for evaluation of the overburden of caregivers of patients with stroke Rev Bras Enferm 2012; 65(5): 839-43.

19. Morais HC, Soares AM, Oliveira AR, Carvalho CM, da Silva MJ, de Araujo TL. Burden and modifications in life from the perspective of caregivers for patients after stroke. Rev Lat Am Enfermagem 2012; 20(5): 944-53.

20. Morimoto T, Schreiner AS, Asano H. Care giver burden and health-related quality of life among Japanese stroke caregivers. Age and Ageing 2003; 32: 218-23.

21. Mollaoglu M, Ozkan-Tuncay F, Kars Fertelli T. Care burden of care givers of stroke patients and related factors. Dokuz Eylul University School of Nursing Electronic Journal 2011; 4(3): 125-30.

22. Berg A, Palomaki H, Lonnqvist J Lehtihalmes M, Kaste M. Depression Among Caregivers of Stroke Survivors Stroke 2005; 36(3): 639-43.

23. Nakipoglu GF, Karamercan A, Mengulluoğlu M, Dal E, Özgirgin N. Comparison of depressive mood findings in geriatric hemiplegic patients and their caregivers. Turkish Journal of Geriatrics 2006; 9(4): 218-21.

24. Han B, Haley WE. Family caregiving for patients with stroke: Review and analysis. Stroke 1999; 30: 1478-85.

25. Dennis M, O'Rourke S, Lewis S, Sharpe M, Warlow C. A quantitative study of the emotional outcome of people caring for stroke survivors. Stroke 1998; 29: 1867-72.

26. Stein PN, Gordon WA, Hibbard MR, Sliwinski MJ. An examination of depression in the spouses of stroke patients. Rehabil Psychol. 1992; 37: 121-30.

27. Miller B, Townsend A, Carpenter E, Montgomery RV, Stull D, Young RF. Social support and caregiver distress J Gerontol B Psychol 2001; 56(4): 249-56.

28. Gerard JM, Landry-Meyer L, Roe JG. Grandparents raising grandchildren: the role of social support in coping with caregiving challenges. The International Journal of Aging 2006; 62(4): 359-83.

29. Clipp EC, George LK. Caregiver needs and patterns of social support. J Gerontol. 1990; 45(3): 102-11.

30. Toseland RW, Rossiter CM. Group Interventions To Support Family Caregivers: A Review and Analysis. The Gerontologist 1989; 29(4): 438-48.

Received: $12 / 01 / 2014$

Accepted in revised form: 09/06/2014

\section{Correspondence author:}

Ali Yavuz Karahan, The Department of Physical Medicine and Rehabilitation of State Hospital of Konya/Turkey, Yunusemre Mh Nurani sk No 1 meram Konya Turkey Zip Code 42090; e-mail: aliyavuzkarahan@outlook.com 